\title{
Effect of Modified Thai Yoga on Energy Cost and Metabolic Intensity in Obese Older Adult Thai Women
}

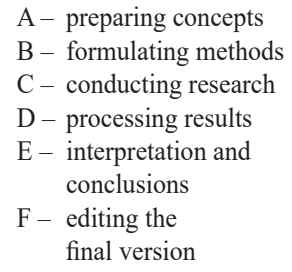

\author{
Waree Widjaja ${ }^{1, A, C-F}$, Kittipun Jitvimolnimit ${ }^{1, B-D}$, Amornpan Ajjimaporn $^{1, C}$, \\ James J. Laskin ${ }^{2, E-F}$ \\ ${ }^{1}$ College of Sports Science and Technology, Mahidol University, Thailand \\ ${ }^{2}$ School of Physical Therapy \& Rehabilitation Sciences, \\ The University of Montana, USA
}

https://doi.org/10.5114/areh.2019.87748

\begin{abstract}
:
Introduction: Obesity is associated with increased risk of chronic diseases. An increasing of physical activity can prevent the obesity and improve the daily life quality in older adults. The purpose of this study was to investigate the effect of modified Thai Yoga on metabolic intensity and energy expenditure in obese older adult Thai women.

Material and methods: Twenty-six participants, aged 56-75 years old, were divided into two groups: non-obese group (NB) and obese group (OB) defined by their body mass index. After 12 sessions of familiarization, the participants performed the modified Thai Yoga exercise program for 60 minutes. Energy expenditure and metabolic variables were measured via open circuit calorimetry.

Results: At the exercise phase, the mean percentage of maximum heart rate for the OB and NB groups were OB: $57.43 \pm 6.32 \%$ and $59.89 \pm 7.94 \%$, respectively. The mean oxygen consumption for the OB group was $7.52 \pm 1.09 \mathrm{ml} / \mathrm{min} / \mathrm{kg}$ as compared to the NB group which was $8.44 \pm 0.90 \mathrm{ml} / \mathrm{min} / \mathrm{kg}$. The mean metabolic equivalent (MET) for the OB group was $2.15 \pm 0.31 \mathrm{MET}$ as compared to $2.41 \pm 0.26 \mathrm{MET}$ for the NB group. Total energy expenditure for the OB and NB groups were $3531.32 \pm 483.47 \mathrm{kcal} /$ day and $3090.82 \pm 387.99 \mathrm{kcal} /$ day, respectively. The mean of activity energy expenditure for the OB group was $1509.65 \pm 498.72 \mathrm{kcal} /$ day and $1393.39 \pm 367.41 \mathrm{kcal} /$ day for the NB group. The difference between all parameters measured from OB and NB groups were significant $(\mathrm{p}<0.05)$.

Conclusions: These results demonstrated that this modified Thai Yoga program provides a well-tolerated light intensity exercise stimulus and could be prescribed as an exercise program for obese older adults.
\end{abstract}

Key words: $\quad$ yoga, obesity, aged women, energy expenditure, metabolic equivalent

\section{Introduction}

The normal aging process affects one's physiological function which results in the decrease of physical performance of daily life activities as well as decreasing skeletal muscle and bone mass, a decline in flexibility and body balance, a lowering of cardiac output and increased blood pressure [1].
Furthermore, the older adult has an increased risk of obesity and the associated chronic diseases such as cardiovascular disease, hypertension and type 2 diabetes mellitus [2]. Moreover, obese individuals tend to be more inactive as compared to normal weight people of the same age. However, the resting energy expenditure in the obese individual is higher than normal weight individuals of the same 
age because of their larger body mass resulting in a higher metabolic rate $[3,4]$.

The ACSM's exercise guidelines for aging adults, recommends that slow paced dynamic movements or static movement exercise can improve and or maintain physical health [5]. It is a well-known fact that Yoga and Tai-Chi are traditional exercise programs which are popular among older adults. Several studies $[6,7,8]$ have reported that Yoga and Tai-Chi provide a low to moderate intensity exercise stimulus which would be suitable for the older adult. Therefore, the three reports are fit well with ACSM's guidelines.

Thai Yoga or Ruesri Dat Ton is an ancient Thai traditional hermit exercise which has been practiced for more than a thousand years [9]. Buranruk, et al. [10] investigated Thai Yoga exercise intensity by comparing it with exhaustive cycling exercise in 14 older adults. This study concluded that Thai Yoga can classified as low to moderate intensity exercise. Furthermore, Noradechanunt, et al. [11] reported that sedentary older adults could improve their physical function and quality of life after a 12-week Thai Yoga training program.

However, many Thai Yoga postures are complicated and involved acrobatic type movements that need high levels of flexibility and muscle strength to perform. In this study, by taking level of difficulty and risk of injury into account the postures were modified by using a chair for providing hand held support for balance and for limiting reach out of base of support. As a result, the modified postures that involves static and dynamic movements are more suitable for obese older adults. The purpose of this study was to investigate the effect of the modified Thai Yoga program on metabolic intensity and energy expenditure in obese and non-obese older adult Thai women.

\section{Materials and Method}

Twenty-six female participants, aged 56-75 years old, participated in this study. They had no prior experience in yoga or tai-chi practice. All were generally healthy; an absence of medical problems such as musculoskeletal injury within the past 6 months, the presence of cardiovascular disease, neuromuscular disease, or disease of inner ear that would increase their risk of a fall.

The participants were informed about the experimental procedures and signed a consent form to participate in this experiment which had been approved by Ethical Committee of Mahidol University. This study was performed in accordance with the ethical standards of the Helsinki Declaration. All potential participants were screened and passed the Physical Activity Readiness Questionnaire or they were cleared by a physician to do the exercise, a health history review, Barthel Activity of Daily Living index ( $>70$ points) and the Berg balance scale ( $>41$ points).

After participants passed the screening procedures, they were divided into two groups by body mass index (BMI). Those ranging from 18.5$23.0 \mathrm{~kg} / \mathrm{m}^{2}$ were placed in the non-obese group (NB) and those between $25.0-29.9 \mathrm{~kg} / \mathrm{m}^{2}$ comprised the obese group (OB). The range of BMI values were set according to Asia-Pacific guidelines [12]. The participants, whom had an estimate peak oxygen consumption value between $24-35 \mathrm{ml} / \mathrm{kg} / \mathrm{min}$, were categorized as fair to excellent classifications by using Heywood's method [13].

\section{Procedures}

On Visit 1, the participants' height, body weight, BMI, blood pressure and percentage of total body fat (\%Body fat) (Omron Karada Scan Body Composition monitor HBF-375, Japan) were measured. A SixMinute Walk Test (6MWT) was administered to estimate peak oxygen consumption ( $\mathrm{VO}_{2}$ peak). The test distance was 15 meters with cone marker place in every 5 meters intervals. Participants were instructed to walk as fast as they could and cover as much distance as they could in safe manner; no jogging or running was allowed. The total distance covered was calculated and used to estimate peak oxygen consumption by the Burr equation [14]: "Estimated $\mathrm{VO}_{2}$ peak $=70.161+(0.023 \times 6 \mathrm{MWT}$ distance $)-(0.276 \times$ body weight $)-6.79-(0.193 \times$ resting heart rate $)-(0.191 \times$ age $)$ " [13]

Before the testing session was undertaken, the participants went through a familiarization program of the modified Thai Yoga exercise program by the instructor to make sure that they could perform the program correctly. All of participants completed the familiarization program of 3 days per week for 4 weeks (12 sessions).

The modified Thai Yoga program was 60 minutes in duration that included a 15-minute warm-up phase (Figure 1a), 30-minute main exercise phase (Figure $1 \mathrm{~b}$ ) and cooldown phase that lasted 15 minutes (Figure 1c). The warm-up phase began 
(a)

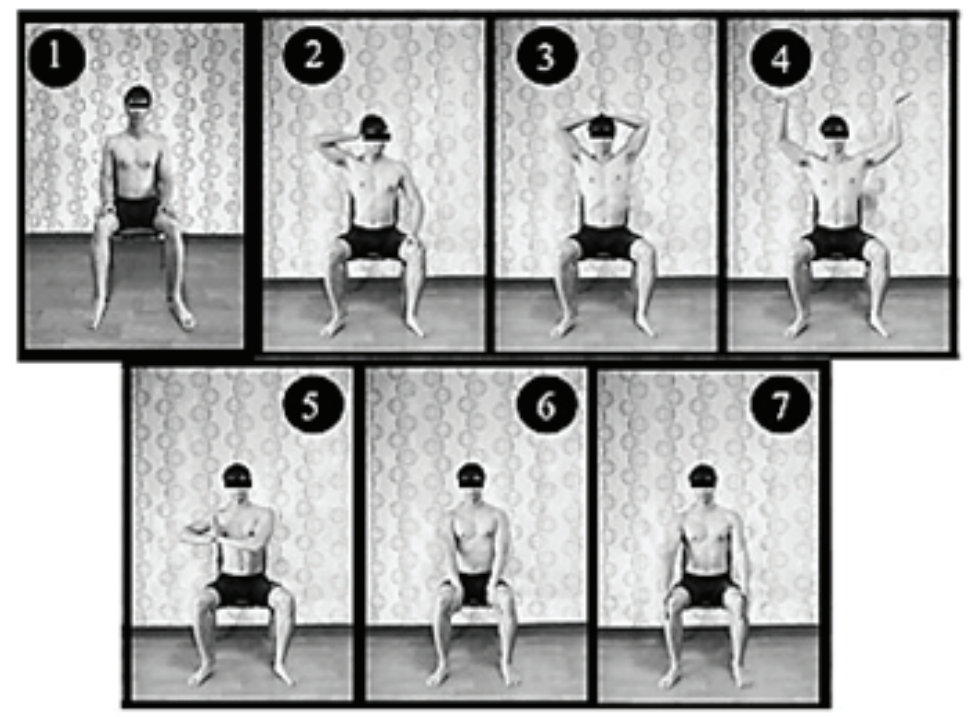

(b)

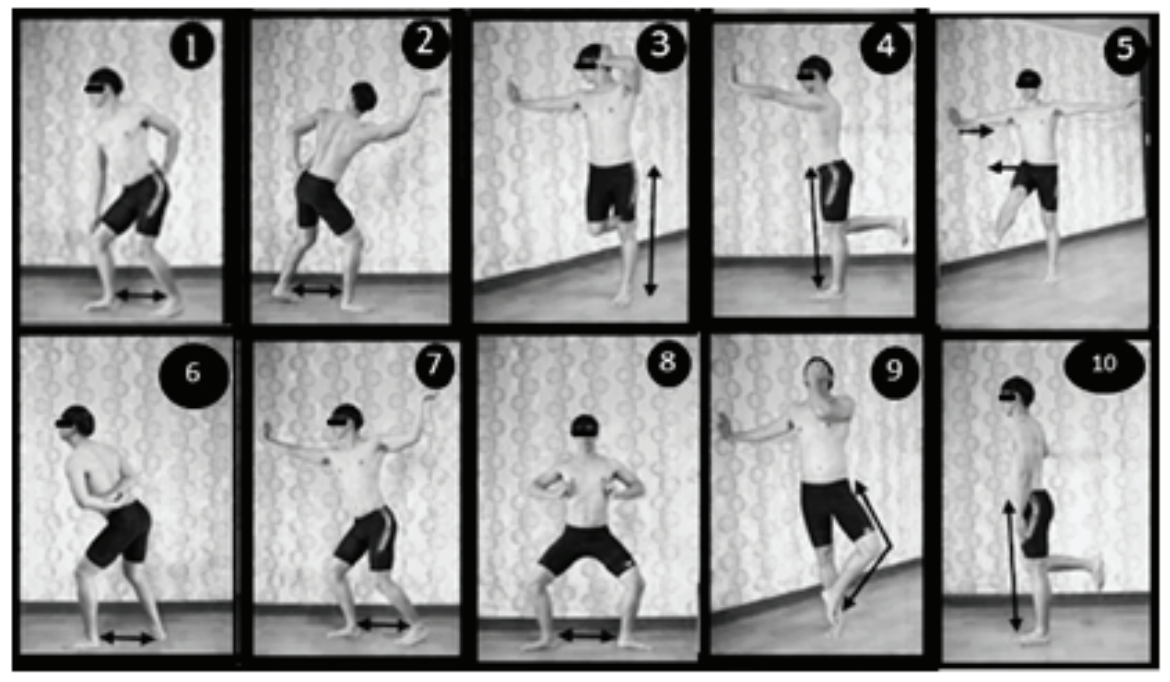

(C)

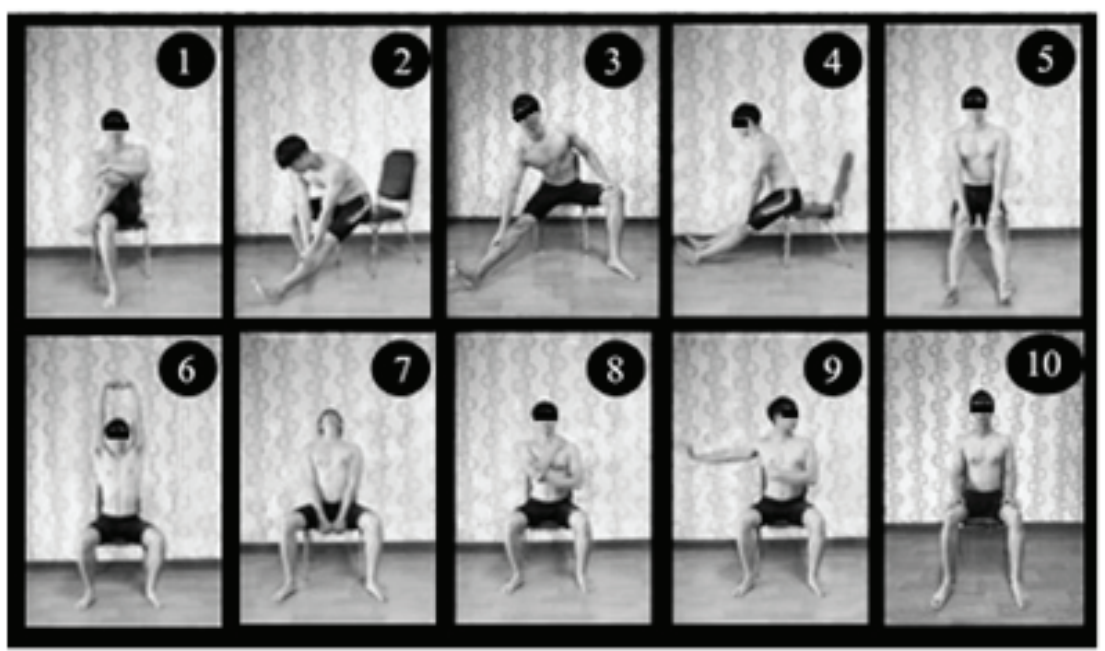

Figure 1. Modified Thai Yoga postures done by a Yoga instructor (a) Warm up, (b) main exercise and (c) cool down, respectively. 
with meditation, then progressed to the warm up postures, each posture was performed on both sides for 2 minutes per posture. The main exercise phase consisted of 10 postures which included both static and dynamic exercise. Each main exercise phase posture was performed on both sides for 3 minutes per posture. The cool down phase, utilized stretching postures and were performed on both sides for 2 minutes per posture and ended with meditation. All of the modified Thai yoga postures were approved by an expert panel (medical doctor, sports scientist, Thai yoga trainer).

On the experimental day, two hours before the participants visited the laboratory, they were instructed to have a normal meal and water intake. The participants donned the indirect calorimeter (Jaeger Oxycon Mobile, Germany) and heart rate monitor (Polar T31, USA). The experimental trial was divided into 3 phases: resting, brisk treadmill walking and the modified Thai Yoga exercise program. For the resting phase, the participants lay supine on a bed for 5 minutes to measure resting energy expenditure (REE). This resting phase was followed by self-selected walking speed on the treadmill with the range of walking speed 2-3 $\mathrm{km} /$ hour for 5 minutes. This was followed by the 60-minute modified Thai Yoga program. All of the metabolic and energy expenditure parameters were collected throughout all 3 phases of the experiment. The measurement points in this study were the average of resting, brisk walking and exercise phases including warm-up postures to the end of main exercise postures. The total energy expenditure (TEE) in each phase was determined by indirect calorimetry and calculated from the total volume of oxygen consumption $\left(\mathrm{VO}_{2}\right)$.

The TEE is the sum of basal energy expenditure (BEE) which is the energy required to maintain the vital body functions, whereas resting energy expenditure (REE) is the energy required to support body's basic metabolic activities that can be $3-10 \%$ higher than BEE, diet-induced thermogenesis (DIT) which is the energy required for digestion, absorption, usage and storage of nutrients. Activity energy expenditure (AEE) is an energy expended beyond the body's basic requirements of REE [15]. For this study the DIT was estimated as $10 \%$ of TEE [16] and AEE was calculated using the equation from DeLany [17] "AEE $=$ TEE $-[$ REE $+0.10 \times$ TEE $]$ ". Finally, the rating of perceived exertion (RPE) was measured by 6-20 Borg's RPE scale after the end of each experimental phase, which estimated the individual's psychological response [18].

Statistical analysis was performed using IBM SPSS version 21 statistical analysis software. Kolmogorov-Smirnov test was performed to test the normality distribution of continuous data and outcome measurement data. The continuous data and outcome measurement data was reported as mean \pm standard deviation. Repeated measures analysis of variance (ANOVA) was used to test the difference of outcome variables (heart rate (HR), $\mathrm{VO}_{2}$, relative volume of oxygen consumed $\left(\mathrm{VO}_{2} /\right.$ $\mathrm{BW})$, total volume of carbon dioxide produced $\left(\mathrm{VCO}_{2}\right)$, relative volume of carbon dioxide produced $\left(\mathrm{VCO}_{2} / \mathrm{BW}\right)$, respiratory exchange ratio (RER), metabolic equivalent (MET), percent of heart rate maximum (\%maxHR), TEE, relative total energy expenditure (TEE/BW), AEE, relative activity energy expenditure (AEE/BW) and RPE) between phases within group and the difference of outcome measurements between NB and OB. Independent sample t-test was used to test the difference of general characteristic measurement. Significant level was set at $\mathrm{p} \leq 0.05$

\section{Results}

Tab. 1. General physical characteristics of the participants.

\begin{tabular}{|l|c|c|}
\hline & $\begin{array}{c}\text { Obese Group } \\
(\mathrm{n}=13)\end{array}$ & $\begin{array}{c}\text { Normal weight } \\
\text { Group }(\mathrm{n}=13)\end{array}$ \\
\hline Age (years) & $64 \pm 5$ & $65 \pm 5$ \\
\hline Height $(\mathrm{cm})$ & $157 \pm 6$ & $158 \pm 6$ \\
\hline Weight $(\mathrm{kg})$ & $65 \pm 6$ & $52 \pm 4 *$ \\
\hline BMI $\left(\mathrm{kg} / \mathrm{m}^{2}\right)$ & $26.43 \pm 1.68$ & $20.99 \pm 1.68 *$ \\
\hline$\%$ Fat $(\%)$ & $37.02 \pm 1.78$ & $33.42 \pm 3.29 *$ \\
\hline Resting heart rate $(\mathrm{bpm})$ & $71 \pm 6$ & $70 \pm 9$ \\
\hline Systolic blood pressure $(\mathrm{mmHg})$ & $124 \pm 13$ & $121 \pm 13$ \\
\hline Diastolic blood pressure $(\mathrm{mmHg})$ & $69 \pm 11$ & $71 \pm 7$ \\
\hline Estimated $\mathrm{VO}_{2}$ peak $(\mathrm{ml} / \mathrm{min} / \mathrm{kg})$ & $30.77 \pm 2.56$ & $34.03 \pm 1.45 *$ \\
\hline
\end{tabular}

Mean $\pm \mathrm{SD}, * \mathrm{p}<0.05$ compared between the obese and the normal weight groups.

The participant characteristics are shown in Table 1. There were no significant differences between NB and OB groups in age, height, resting HR, systolic blood pressure and diastolic blood pressure. However, as expected here was a significant difference $(\mathrm{p}<0.05)$ between the NB and $\mathrm{OB}$ groups in weight, BMI, \% Bodyfat and estimated $\mathrm{VO}_{2}$ peak. 
HR

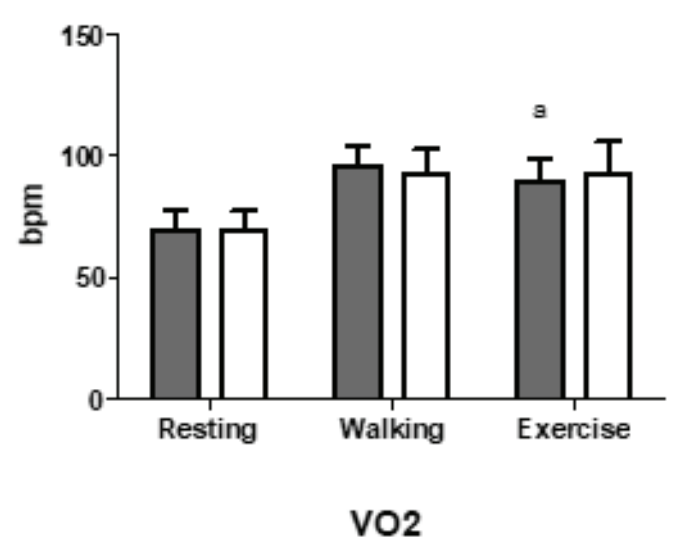

RER

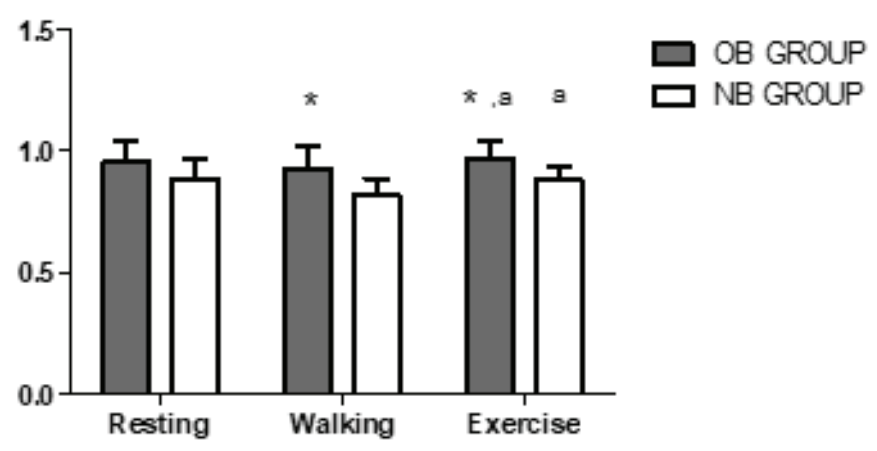

VO2/BW

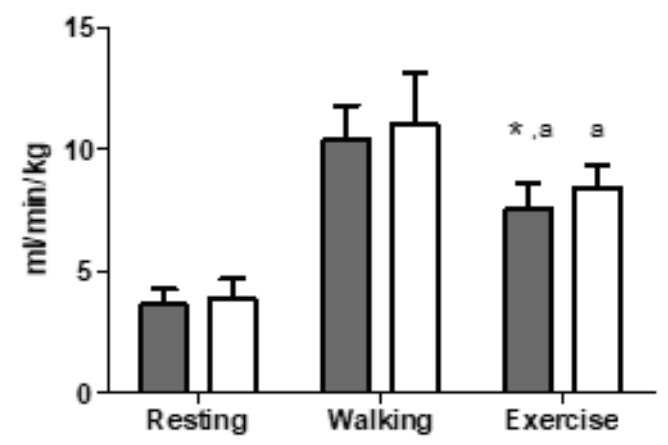

VCO2/BW

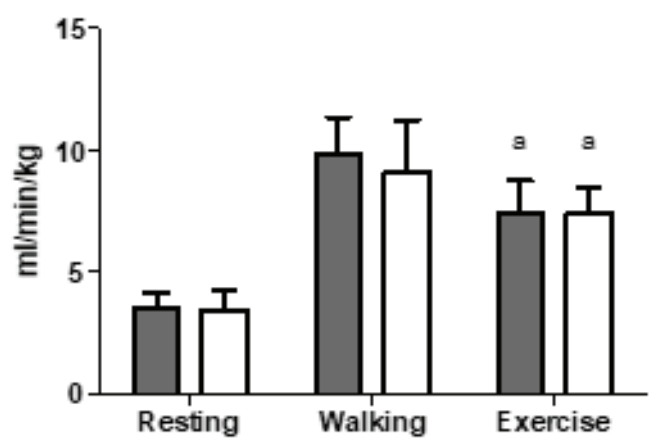

Fig. 2 Heart rate and metabolic responses to the modified Thai Yoga exercise program. Mean \pm SD, obese group (OB), non-obese group (NB), heart rate (HR), respiratory exchange ratio (RER), total oxygen consumption $\left(\mathrm{VO}_{2}\right)$, relative osygen consumption $\left(\mathrm{VO}_{2} / \mathrm{BW}\right)$, total carbon dioxide rpoduction $\left(\mathrm{VCO}_{2}\right)$, relative carbon dioxide production $\left(\mathrm{VCO}_{2} / \mathrm{BW}\right)$.

$\cdot \mathrm{p}<0.05$ represents a significant difference at resting, treadmill walking, and exercise phase between groups, ${ }^{2} \mathrm{p}<0.05$ represents a significant difference: treadmill walking vs exercise phase inboth groups.

Figure 2 shows that while there were no significant differences in HR between groups in any of the 3 phases, there was significant difference between treadmill walking and exercise phases in OB group. There were also significant differences in $\mathrm{VO}_{2}$ between and within groups while walking on the treadmill and during the modified Thai yoga exercise program. As expected the $\mathrm{VO}_{2} / \mathrm{BW}$ was significantly greater in the NB group during the exercise phase.
Figure 3 shows the mean MET values were significantly lower during the modified Thai yoga than during the self-selected brisk walking pace on the treadmill for both groups. During the modified Thai yoga activity, the MET level was significantly greater for the NB $(2.41 \pm 0.26$ MET) as compared to the OB $(2.15 \pm 0.31$ MET). Interestingly the \%maxHR during Thai yoga was significant lower for the $\mathrm{OB}$ as compared to the treadmill walking activity. Regarding absolute TEE 
MET

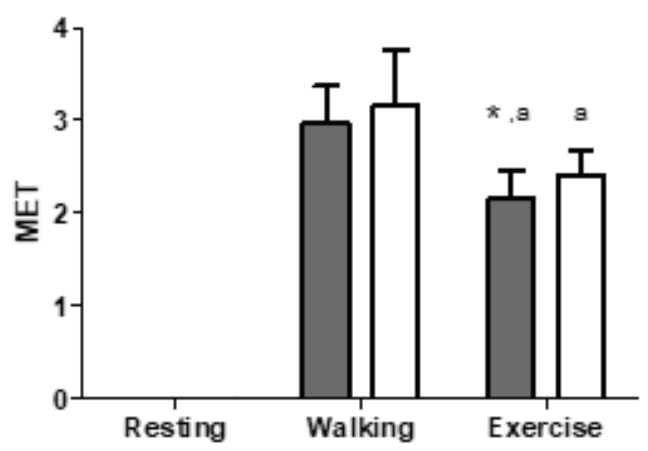

TEE

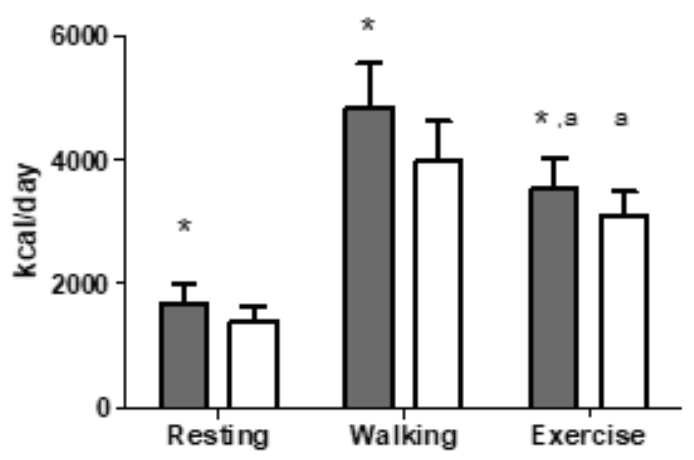

AEE

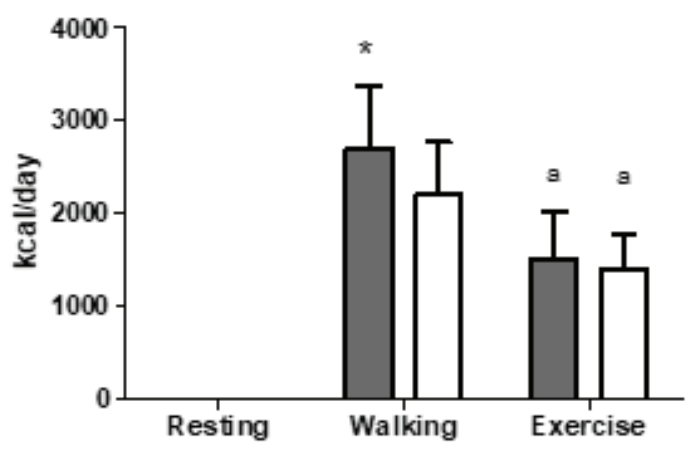

$\% \operatorname{maxHR}$

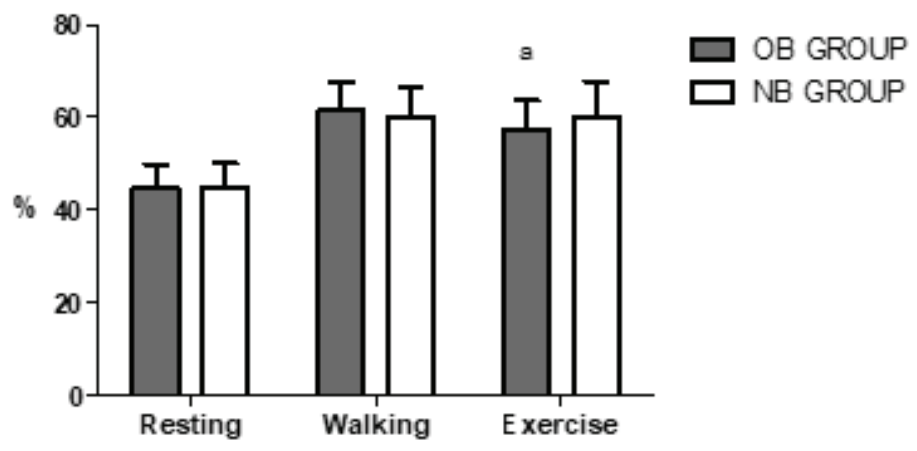

TEE/BW

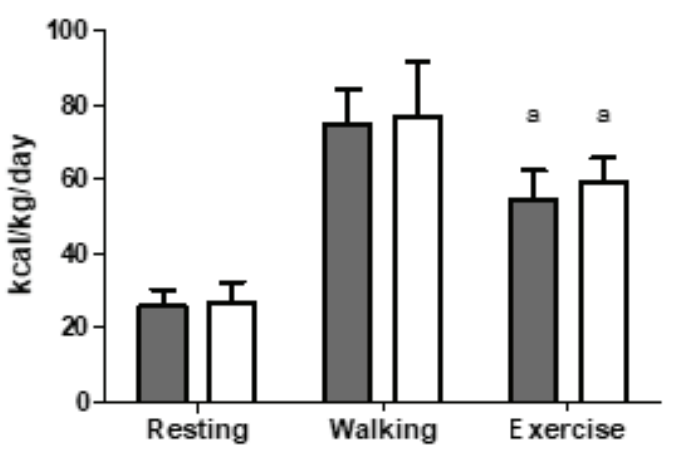

AEE/BW

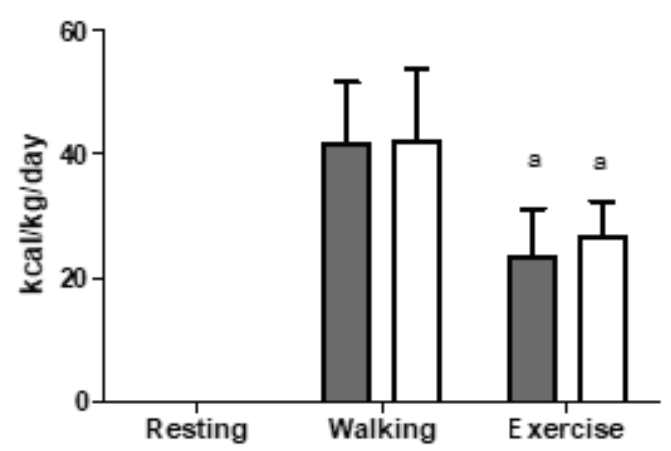

Fig. 3 Impact of the modified Thai Yoga exercise program on energy expenditure.

Mean $\_$SD, obese group $(\mathrm{OB})$, non-obese group (NB), metabolic equivalent (MET), percent of maximal heart rate (\%maxHR), absolute total energy expenditure (TEE), relative total energy expenditure (TEE/BW), total activity energy expenditure (AEE), relative activity energy expenditure (AEE/BW).

$* \mathrm{p}<0.05$ represents a signific ant difference at resting, treadmill walking and exercise phases between groups, ${ }^{\text {a }} \mathrm{p}<0.05$ represented a significant difference: treadmill walking vs exercise in both groups.

and AEE the values were significantly reduced for the OB during the Thai yoga activity as compared to treadmill walking. Interestingly, there were no significant relative differences between groups while performing the modified Thai yoga program.
Regarding RPE, there were no significant difference between groups. However, the OB group found the modified Thai yoga program significantly harder as compared to walking on the treadmill at their self-selected brisk walking pace. 


\section{Discussion}

Thai yoga or Ruesri Dat Ton is an alternative exercise option of a traditional Thai activity that could be used to improve physical performance in elderly Thai [10]. This study, utilized modified Thai Yoga postures to safely promote this traditional Thai activity in obese older women. Our results showed that the intensity of the modified Thai yoga was $57.43 \pm 6.32 \%$ maxHR in the OB group, and $59.89 \pm 7.94 \%$ maxHR in the NB group. There was no significant difference between groups. This suggests, support by the observed MET levels (2.15 \pm 0.31 MET and $2.41 \pm 0.26$ MET for the OB and NB respectively) that this modified Thai yoga program provides a light intensity stimulus. This conclusion is supported by the classification of "light activity" by the American College of Sports Medicine's guidelines as being and activity with a NEAT level of $<3$ or at 50-64\% maxHR (5). Furthermore, RPE, the subjective evaluation for psychological response of exercise intensity also showed the level of feeling to evaluate the exercise intensity as "light intensity" in both groups.

To further support the suggestion that the metabolic intensity of modified Thai Yoga is a "light exercise intensity" activity, we found that the HR, $\%$ maxHR and MET of modified Thai Yoga in the OB group was significantly lower compared with those values observed during the self-selected brisk walk on the treadmill. One potential explanation, the participants were free to performed each posture at their own intensity. Secondly, the modified Thai Yoga postures performed included both static exercise postures for balancing and muscle strength while the dynamic exercise postures for cardiovascular function. Therefore, the intensity in modified Thai Yoga appears to be less than brisk walking.

Although, in an earlier study, Ravussin et. al., [19] suggested that the obese have a "slowed metabolism", however, over the past 30 years the literature has consistently reported contradictory findings, proposing that the obese actually have a higher absolute energy expenditure than their nonobese counterparts $[17,20,21]$. This pattern was consistent with the findings of our study with the absolute TEE and AEE in the OB being higher than those in NB [20,21]. However, if we adjusted TEE and AEE by dividing by body weight, there was not a significant difference between groups.

Buranruk [10], examined the metabolic intensity of Thai Yoga exercise by studying 14 older adults with a BMI of $>25.75 \mathrm{~kg} / \mathrm{m}^{2}$ (obese). They performed $30 \mathrm{~min}$ of Thai Yoga that including 8 sitting postures, 4 standing postures and 3 lying postures. All of the postures were static postures. The results of this study provided values of metabolic intensity that were lower than our present study. However, this may be explained by the exclusive use of static postures in Buranruk's study. It can be safely assumed that the addition of dynamic postures in our study induced the higher energy expenditure values that were observed.

While only providing a light intensity stimulus, a program the includes modified Thai yoga postures can provide an alternative exercise option for both obese and normal weight elderly individuals. Furthermore, it is worth mentioning that after doing the exercises, the obese older participants did not suffer from any adverse medical events. Therefore, the modified Thai yoga is a safe intervention for older adults. As for further studies, modifications to increase the exercise stimulus as well as potential benefits to body balance, muscle strength, bone health and motor skills [22] should be investigated.

\section{Conclusions}

Modified Thai Yoga is a safe and potentially effective exercise intervention for both obese and normal weight older women. While the metabolic intensities and energy costs observed in this study demonstrated that this intervention provides a light intensity stimulus, it could be an optional exercise program for obese and normal weight older adults alike. 


\section{References:}

1. Boss GR, Seegmiller JE. Age-related physiological change and their clinical significance, In Geriatric Medicine. West J Med. 1981 Dec;135(6):434-40.

2. Sahin TT. Physiologic changes in obesity and patient preparation for bariatric surgery. Laparosc Endrosc Surg Sci. 2016;23(3):63-71

3. Manini TM. Energy expenditure and aging. Ageing Res Rev. 2010 Jan; 9(1):1-11.

4. Siervo M, Oggioni C, Lara J, Celis-Morales C, Mathers JC, Battezzati A. et al. Age-related change in resting energy expenditure in normal weight, overweight and obese men and woman. Maturitas. 2015;Apr 80(4):406-13.

5. American College of Sports Medicine. ACSM's Guideline for exercise testing and prescription. 9th ed: Wolters Kluwer Health, Lippincott Williams \& Wilkins; 2013.

6. Larson-Meyer DE. A systematic review of the energy cost and metabolic intensity of yoga. Med Sci Sports Exerc. 2016 Aug;48(8):1558-69.

7. Danucalov MAD, Simoes RS, Kozasa EH, Leite JR. Cardiorespiratory and metabolic changes during yoga sessions: The effect of respiratory exercises and meditation practices. Appl Psychophysiol Biofeedback. 2008 Jun;33(2):77-81.

8. Chao YFC, Chen SY, Lan C, Lai JS. The cardiorespiratory response and energy expenditure in Tai-Chi-Qui-Gong. Am J Chin Med. 2002; 30(4):451-61.

9. Well D. Ruesri Dat Ton: The Thai Hermit's Exercises. Yoga Mimamsa. 2012 Apr; 44(2):130-47.

10. Buranruk O, La Grow S, Ladawan S, Makarawate P, Suwanich T, Leelayuwat N. Thai yoga as an appropriate alternative physical activity for older adults. J Complement Integr Med 2010; 7(1):1-14.

11. Noradechanunt C, Worsley A, Groeller H. Thai Yoga improves physical function and well-being in older adults: A randomized controlled trial. J Sci Med Sport. 2017 May; 20(5):494-501.

12. Lim JU, Lee JH, Kim JS, Hwang YI, Kim TH, Lim SY, Yoo KH, Jung KS, Kim YK, Rhee CK. Comparison of World Health Organization and Asia-Pacific body mass index classifications in COPD patients. Int. J. COPD 2017 Aug;12:2465-75.

13. Heywood V. Advanced fitness assessment and exercise prescription, 5th ed, Champaign, IL: Human Kinetics; 2006.

14. Burr JF, Bredin SSD, Faktor MD, Warburton DER. The 6- minute walk test as a predictor of objectively measured aerobic fitness in healthy working-aged adults. Phys Sportsmed. 2011 May;39(2):133-9.

15. Carneiro IP, Elliott SA, Siervo M, Padwal R, Bertoli S, Batterzzati A. et al. Is obesity associated with Altered Energy Expenditure? Adv Nutr. 2016 May;7(3):476-87.

16. Levine JA. Non-exercise activity thermogenesis (NEAT). Best Pract Res Clin Endocrinol Metab. 2002 Dec;16(4):679-702.

17. DeLany JP, Kelley DE, Hames KC, Jakicic JM, Goodpaster BH. High energy expenditure masks low physical activity in obesity. Int J Obes (Lond). 2013 Jul;37(7):1006-11.

18. Borg GA. Psychophysical bases of perceived exertion. Med Sci Sport Exerc. 1982; 14(5): 377-81.

19. Ravussin E, Lillioja S, Knowler WC, Christin L, Freymond D, Abbott WG, et al. Reduced rate of energy expenditure as a risk factor for body- weight gain. N Eng J Med. 1988 Feb;318:467-72.

20. LeCheminant JD, Heden T, Smith J, Covington NK. Comparison of energy expenditure, economy, and pedometer counts between normal weight and overweight or obese women during a walking and jogging activity. Eur J Appl Physiol. 2009 Jul; 106: $675-82$.

21. Johannsen DL, Welk GJ, Sharp RL, Flakoll PJ. Differences in daily energy expenditure in lean and obese women: The role of posture allocation. Obesity. 2008 Jan;16:34-9.

22. Świst A, Graff K, Chałubińska D, Zielińska A, Łukaszewska A. The effects of interventions implemented during a rehabilitation camp on changes in motor skills parameters and body mass in patients with simple obesity. Adv Rehab 2017;3: 5-15. 